\title{
Review Article \\ Power Law and Entropy Analysis of Catastrophic Phenomena
}

\author{
J. A. Tenreiro Machado, ${ }^{1}$ Carla M. A. Pinto, ${ }^{2}$ and A. Mendes Lopes ${ }^{3}$ \\ ${ }^{1}$ Department of Electrical Engineering, Institute of Engineering, Polytechnic of Porto (ISEP), \\ Rua Dr. António Bernardino de Almeida, 431, 4200-072 Porto, Portugal \\ ${ }^{2}$ Institute of Engineering, Polytechnic of Porto (ISEP) and Center of Mathematics of the University of Porto, \\ Rua Dr. António Bernardino de Almeida, 431, 4200-072 Porto, Portugal \\ ${ }^{3}$ UISPA, IDMEC-Polo FEUP, Faculty of Engineering, University of Porto, Rua Dr. Roberto Frias, \\ 4200-465 Porto, Portugal
}

Correspondence should be addressed to J. A. Tenreiro Machado; jtm@isep.ipp.pt

Received 17 September 2012; Accepted 13 December 2012

Academic Editor: József Kázmér Tar

Copyright (C) 2013 J. A. Tenreiro Machado et al. This is an open access article distributed under the Creative Commons Attribution License, which permits unrestricted use, distribution, and reproduction in any medium, provided the original work is properly cited.

Catastrophic events, such as wars and terrorist attacks, tornadoes and hurricanes, earthquakes, tsunamis, floods and landslides, are always accompanied by a large number of casualties. The size distribution of these casualties has separately been shown to follow approximate power law (PL) distributions. In this paper, we analyze the statistical distributions of the number of victims of catastrophic phenomena, in particular, terrorism, and find double PL behavior. This means that the data sets are better approximated by two PLs instead of a single one. We plot the PL parameters, corresponding to several events, and observe an interesting pattern in the charts, where the lines that connect each pair of points defining the double PLs are almost parallel to each other. A complementary data analysis is performed by means of the computation of the entropy. The results reveal relationships hidden in the data that may trigger a future comprehensive explanation of this type of phenomena.

\section{Introduction}

Power laws (PLs) have been widely reported in the modeling of distinct phenomena and have been associated with long memory behavior, self-similarity, fractal structures and fractional calculus. In [1], for example, PLs are interpreted as a manifestation of the long memory property of systems with fractional dynamics and, in [2], several complex systems exhibiting PL behavior are analysed in the perspective of fractional dynamics. Fractional calculus and PLs are used in $[3,4]$ to model the dynamics of financial markets. In [5] the complexity of the human body is characterized through fractal measures and its dynamics described by means of fractional calculus.

Catastrophic events are characterized by a huge severity, usually defined by a large number of casualties. By catastrophic events, we mean wars, terrorist attacks, tornadoes, earthquakes, floods, and landslides. The distribution of the number of casualties in these events is proved to be a PL [6-12].
PL distributions were first mentioned in 1896, when Pareto described the distribution of income [13]. Pareto proved that the relative number of individuals with an annual income larger than a certain value $x$ was proportional to a power of $x$. This has been known by Pareto distribution. After this work, Auerbach [14] demonstrated an analogous result for city size distributions. Ranking cities from 1 to $n$, with the city with bigger population ranked as 1 , Auerbach demonstrated that the product of cities populations by their ranks was approximately constant, for a given territory. Estoup [15] and Zipf [16, 17] applied PLs to words frequencies in texts. They found that there are words that are used more often than others and the distribution of word frequencies follows a PL. Zipf [17] described the distribution of city sizes by a Pareto distribution.

Often, to show that a certain data set follows a PL distribution, researchers depict a plot of the size versus frequency of the event studied. In logarithmic scales, they obtain a straight line with negative slope. In the case of 
the Pareto distribution, the behavior is exactly linear, and is given by

$$
\ln (P[X \geq x])=\ln C-\ln \tilde{\alpha}-\tilde{\alpha} \ln x,
$$

where $X$ is a random variable following a PL distribution, $\widetilde{\alpha}>0, \widetilde{C}=C / \widetilde{\alpha}>0$. In these distributions, the tail falls asymptotically according to the value of $\widetilde{\alpha}$, translating in heavy tails, comparatively to other distributions. Zipf's law is a special case of the Pareto's law, with coefficient $\widetilde{\alpha}=1$. Relevant reviews on PL distributions can be found in [18-20].

In many cases a single PL holds for the entire range of the random variable that represents the system. In other cases, the statistical distribution is better described by multiple PLs [21]. In such cases, different PLs, characterized by distinct PL parameters, fit, more adequately, the real data. Double PL behaviors have been pointed out by others in different phenomena. For example, in [22] many instances are shown of two PLs expressed by means of a generalized beta distribution function and, in [23], the double PL behavior is explicitly studied in the frequency of words in texts. Moreover, beyond the ranking problem, two PLs are manifested in other type of problems such as in turbulence, earth magnetic pole fluctuations, paleolake sedimentation density subject to volcanism, and avalanche distributions, to mention a few [24].

In this paper, we analyze the statistical distributions of the number of victims caused by catastrophic phenomena and find double PL behavior. Moreover, we plot the PL parameters, corresponding to several events, and observe an interesting pattern in the charts, where the lines that connect each pair of points defining the double PLs are roughly parallel to each other. Then, a complementary data analysis is performed by means of the computation of the Shannon entropy. The results reveal relationships hidden in the data that may trigger a future comprehensive explanation of this type of phenomena.

Bearing these ideas in mind, this paper is organized as follows. In Section 2, the results found in the literature concerning PLs and casualties in natural and human-made disasters are summarized. Section 3 analyses the PL behavior of catastrophic phenomena using data from real disasters. In Section 4 several entropy measures are used to characterize the data. Finally, in Section 5, the main results and conclusions of this paper are discussed.

\section{Brief Review of PLs in Catastrophic Occurrences}

Patterns seen in wars, terrorist attacks, tornadoes, earthquakes, landslides, floods, and other severe occurrences have been at close attention by various researchers [6-12, 25-28]. Many attentive explanations have arisen in the literature. Nevertheless, a complete understanding of these patterns is a complex task. Important and intricate political, geographical, historical, and, even cultural, factors oppose to a better understanding. Predicting the number of casualties in natural or human-made disasters is extremely important in developing predisaster strategies. Aspects like rationalization of medical supplies and food, gathering emergency teams, organize shelter spaces, amongst others, have to be dealt with, in order to minimize the damage.

A PL behavior is indicative of a particular property of a system, it indicates that the size of an event is inversely proportional to its frequency. In this sense, large casualties are associated with low frequency phenomena, and more frequent events are less harmful in terms of preserving human lives [7, 25]. Examples of phenomena with low probability and huge casualties are the two world wars (WWs), high magnitude earthquakes, strong tornadoes, huge tsunamis, and amongst others.

In 1948, Richardson [7], analyzed domestic and international cases of violence, in the period from 1820 to 1945 . He distributed the cases, according to casualties measured in powers of 10, into five categories. The two WWs were classified in the highest category. In a later work [25], the same author showed that if the frequency of an occurrence decreased by a factor close to three, then the number of casualties increased by a power of 10 .

Guzzetti [26] considers landslide events in specific periods in different countries, such as Italy, Canada, Alps, Hong Kong, Japan, and China. He shows that the plot of the cumulative distribution function of the number of landslide events versus the number of casualties is well approximated by a straight line. This result suggests a PL distribution of the data.

Cederman [11] followed Richardson's work [7, 25]. He used data from the Correlates of War (COW) Project [29], focusing on interstate wars. He computed the cumulative relative frequency of war size and showed that it obeyed a PL. The author proposed a self-organized critical dynamical system, that replicated the PL behavior seen in real data. Its model allowed conflict to spread and diffuse, potentially over long periods of time, due to the quasi-parallel execution.

In 2005, Jonkman [27] studied the distribution of killings in global events, focusing on the number of human deaths caused by three types of floods (river floods, flash floods, and drainage issues), between January 1975 and June 2002. The author plotted the global frequency of events with $N$ or more deaths versus $N$. He observed a PL behavior for earthquakes but not for flood data. Becerra et al. [30] use the same data set as Jonkman [27], but consider all disasters combined, both globally and disaggregated by continent. They obtained straight-line log-log plots for all disasters combined. The slopes of the casualties PL distributions were smaller than those for modern wars and terrorism. The explanation for this remained an open question. Another unsolved issue was the existence of PL behavior in combined disasters and not in individual disasters, such as floods. Here it is worth mentioning that casualties in earthquakes verified a PL distribution $[6,27,30]$.

Johnson et al. [28] suggested a microscopic theory to explain similarity in patterns of violence, such as war and global terrorism. The similarity was observed regardless of 
underlying ideologies, motivations, and the terrain in which events occurred. The authors introduced a model where the insurgent force behaved as a self-organizing system, which evolved dynamically through the continual coalescence and fragmentation of its constituent groups. They analyzed casualties' patterns arising within a given war, unlike previous studies that focused on the total casualty figure for one particular war $[7,11,25,31]$. A PL behavior fitted well the data not only from Iraq, Colombia, and non-G7 terrorism, but also with data obtained from the war in Afghanistan. The PL parameter for Iraq, Colombia, and Afghanistan was (close to) $\widetilde{\alpha}=2.5$. This value of the coefficient equalized the coefficient value characterizing non-G7 terrorism. In the literature, the PL parameter value was $\widetilde{\alpha}=2.51$ for non-G7 countries [32] and $\widetilde{\alpha}=1.713$ for G7 countries. This result suggested that PL patterns would emerge within any modern asymmetric war, fought by loosely-organized insurgent groups.

In 2006, Bogen and Jones [33] treated the severity of terrorist attacks in terms of deaths and injured. They applied a PL distribution to victim/event rates and used the PL to predict mortality due to terrorism, through the year 2080. Authors claimed that these PL models could be used to improve strategies "to assess, prevent and manage terror-related risks and consequences".

Clauset et al. [34] studied the frequency and the number of casualties (deaths and injuries) of terrorist attacks, since 1968. They observed a scale-invariance behavior, with the frequency being an inverse power of the casualties. This behavior was independent of the type of weapon, economic development, and distinct time scales. The authors presented a new model to fit the frequency of severe terrorist attacks, since previous models in the literature failed to produce the heavy tail in the PL distribution. Their model assumed that the severity of an occurrence was a function of the execution plan, and that selection tools were better suited to model competition between states and nonstate actors. Finally, researchers claimed that periodicity was a common feature in global terrorism, with period close to roughly 13 years.

Bohorquez et al. [12] studied the quantitative relation between human insurgency, global terrorism and ecology. They introduced a new model to explain the size distribution of casualties or the timing of within-conflict events. They considered insurgent populations as self-organized groups that dynamically evolved through decision-making processes. The main assumptions of the model were (i) being consistent with work on human group dynamics in everyday environments, (ii) having a new perception of modern insurgencies, as fragmented, transient, and evolving, and (iii) using a decision-making process about when to attack based on competition for media attention. Authors applied a PL distribution to Iraq and Colombia wars, with parameter value close to $\widetilde{\alpha}=2.5$. A coefficient value of $\widetilde{\alpha}=2.5$ was in concordance with the coefficient value of $\widetilde{\alpha}=2.48 \pm 0.07$ obtained by Clauset et al. [34] on global terrorism. A PL fit to Spanish and American Civil wars revealed a PL parameter value smaller (around $\widetilde{\alpha}=1.7$ ). Authors claimed that their model suggested a remarkable link between violent and nonviolent human actions, due to its similarity to financial market models.

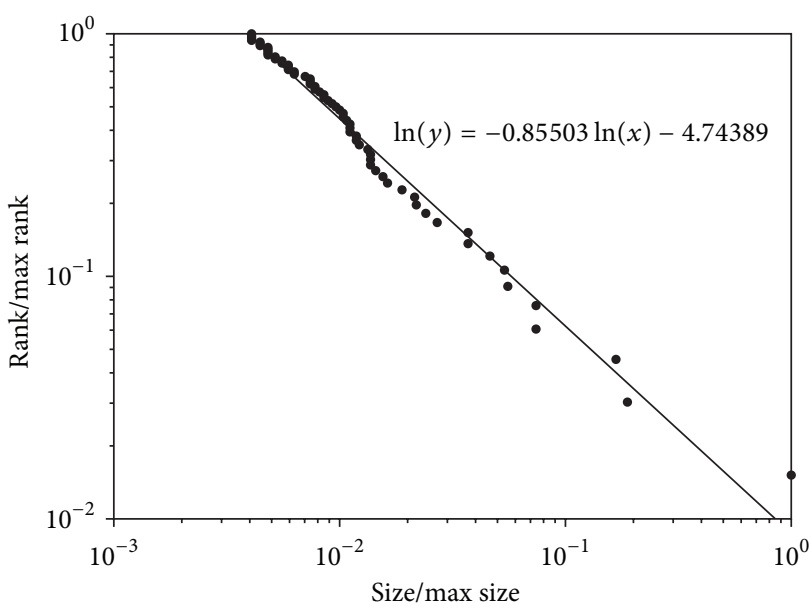

FIGURE 1: Rank/frequency log-log plot corresponding to the distribution of casualties caused by industrial accidents in Central/South America over the period 1900-2011 $(\min$ size $=10 ; \max$ size $=2700$; $\max \operatorname{rank}=66$ ).

\section{Power Law Behavior in Catastrophic Phenomena}

In this section we investigate the statistical distributions of random variables that represent the number of human casualties in several human-made and natural hazards.

Data from the EM-DAT International Disaster Database (http://www.emdat.be/) and the Global Terrorism Database (GTD) (http://www.start.umd.edu/gtd/) are analyzed. The EM-DAT database contains information on over than 18000 worldwide natural and technological disasters, from 1900 to present. The EM-DAT is maintained by the Centre for Research on the Epidemiology of Disasters (CRED) at the School of Public Health of the Université Catholique de Louvain, located in Brussels, Belgium [35]. The GTD database is an open-source database that includes information on more than 98000 worldwide terrorist attacks, from 1970 up to 2010 [31].

PLs are observed in several natural and man-made systems. Examples of single and double PLs in real data are given in Figures 1 and 2, respectively. The former represents the complementary cumulative distribution of the severity of industrial accidents in Central/South America over the period 1900-2011. The adopted measure to quantify the severity of an event is the total number of fatalities. The depicted graph corresponds to a rank/frequency log-log plot. To construct the graph, we first sort the data (i.e., the accidents) in decreasing order according to their severity, and number them, consecutively, starting from one [36]. Then a normalization of the values is carried out, meaning that the number of fatalities ( $x$-axis) is divided by the corresponding highest value, and the rank ( $y$-axis) is divided by the rank of the smallest event. Finally, PLs are adjusted to the data using a least squares algorithm. All the log-log plots presented in this paper are made following this procedure.

Figure 2 corresponds to the distribution of casualties caused by earthquakes in Central/South America in 


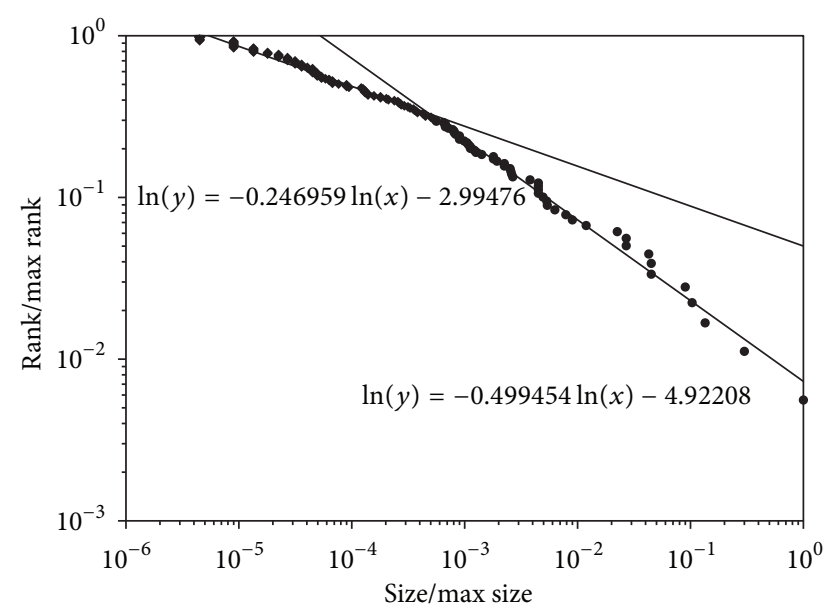

FIGURE 2: Rank/frequency log-log plot corresponding to the distribution of casualties caused by earthquakes in Central/South America over the period 1900-2011 ( $\min$ size $=1 ; \max$ size $=222570$; $\max \operatorname{rank}=179$ ).

the period 1900-2011, representing one event that can be approximated by a double PL.

As can be seen in Figure 1, a single PL (SPL) with parameters $(\widetilde{C}, \widetilde{\alpha})=(0.0087,0.8550)$ fits to the data. The distribution depicted in Figure 2 is better approximated by a double PL (DPL) with parameters $\left(\widetilde{C}_{1}, \widetilde{\alpha}_{1}\right)=(0.0500,0.2470)$ and $\left(\widetilde{C}_{2}, \widetilde{\alpha}_{2}\right)=(0.0073,0.4995)$. The change in the behavior occurs at the relative value of $x=0.000539$, approximately.

We analyzed the data available at the EM-DAT database in terms of disaster type $\left(\mathrm{DT}_{j}\right)$ and disaster location $\left(\mathrm{DL}_{k}\right)$, $j=1, \ldots, 11$ and $k=1, \ldots, 6$ categories, respectively: $\mathrm{DT}_{j}=\{$ Drought, Earthquake, Epidemic, Extreme temperature, Flood, Industrial accident, Mass movement wet, Storm, Transport accident, Volcano, Wildfire $\} ; \mathrm{DL}_{k}=\{$ Africa, North America, Central \& South America, Europe (including Russia), Asia (not including SE Asia), Oceania (including SE Asia) $\}$. The period of analysis was 1900-2011 for every case. The total number of combinations (location/type) is $11 \times$ 6. Nevertheless, for 14 cases, there is insufficient data to compute reliable statistical distributions. For all cases, taking the number of casualties as the variable of interest, we obtain statistical distributions that can be approximated by either a SPL (16 cases) or a DPL (36 cases), similar to the ones depicted in Figures 1 and 2.

In Figure 3 we depict the locus of the parameters $\left(\widetilde{C}_{i}, \widetilde{\alpha}_{i}\right), i=1,2$, corresponding to the analyzed cases. As can be seen, an interesting pattern emerges, where the lines that connect the pairs of points that characterize the DPLs have identical orientation. This geometrical pattern reflects a relationship between the two parts of the DPL distributions (DPL1-part closer to the head; and DPL2- part closer to the tail). Besides the observation that $\widetilde{\alpha}_{2}>\widetilde{\alpha}_{1}$, in all cases, further investigation on the reason for this behavior is needed.

We pursued our study with the analysis of the GTD database. First, the events associated to human casualties were grouped by year $\left(Y_{r}\right)$ starting in 1980 up to 2010

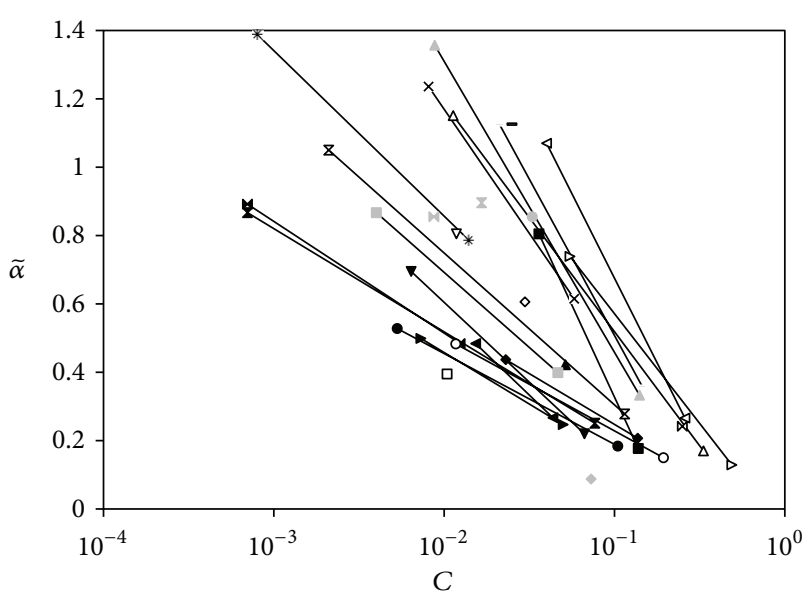

FIGURE 3: Locus of the parameters $\left(\widetilde{C}_{i}, \widetilde{\alpha}_{i}\right), i=1,2$, that characterize the PLs corresponding to the number of casualties in certain combinations of disaster type/location, $\mathrm{DT}_{j} / \mathrm{DL}_{k}$.

(except 1993, because there is no data available): $Y_{r}=$ $\{1980, \ldots, 2010\} \backslash\{1993\}, r=1, \ldots, 30$. We found that all the statistical distributions can be approximated by DPLs. In Figures 4 and 5 , the time evolution of the parameters of the DPLs $\left(\widetilde{C}_{i}, \widetilde{\alpha}_{i}\right), i=1,2$, is shown. Regarding the parameters $\widetilde{C}_{i}$, it can be seen that they have identical behavior, although $\widetilde{C}_{2}$ varies more than $\widetilde{C}_{1}$ and is always smaller than it.

With respect to $\widetilde{\alpha}_{i}$, we have a similar evolution but, in this case, the parameter $\widetilde{\alpha}_{2}$ is always greater than $\widetilde{\alpha}_{1}$. As severe terrorist attacks correspond to points closer to the tail of the distribution, DPL2, which is characterized by a larger $\widetilde{\alpha}$, this means that those events are more similar between each other than the smaller events (that correspond to DPL1).

To complement the analysis with respect to the date of the occurrences, the parameters $\left(\widetilde{C}_{i}, \widetilde{\alpha}_{i}\right)$ of the PLs, corresponding to $Y_{r}$, were plotted (Figure 6). As can be seen, a pattern similar to the described previously (Figure 3 ) is observed.

We have also studied the distributions of the casualties in terrorist attacks, occurred in the period 1970-2010, but with respect to other criteria, namely, the type of used weapon $\left(W_{i}\right)$, region where the event took place $\left(R_{j}\right)$, target $\left(T_{k}\right)$, and type of attack $\left(A_{l}\right)$. Each criterion was then divided into $i=1, \ldots, 6, j=1, \ldots, 13, k=1, \ldots, 19$, and $l=$ $1, \ldots, 8$ categories, respectively: $W_{i}=\{$ Chemical, Explosives, Firearms, Incendiary, Melee, Vehicle $\} ; R_{j}=\{$ Australasia \& Oceania, Central America \& Caribbean, Central Asia, East Asia, Eastern Europe, Middle East \& North Africa, North America, South America, South Asia, Southeast Asia, SubSaharan Africa, USSR \& Newly Independent States (NIS), Western Europe $\} ; T_{k}=\{$ Airports \& Airlines, Business, Educational Institution, Food or Water Supply, Government (Diplomatic), Government (General), Journalists \& Media, Maritime, Military, NGO, Police, Private Citizens \& Property, Religious Figures/Institutions, Telecommunication, Terrorists, Tourists, Transportation, Utilities, Violent Political Party $\} ; A_{l}=\{$ Armed Assault, Assassination, 


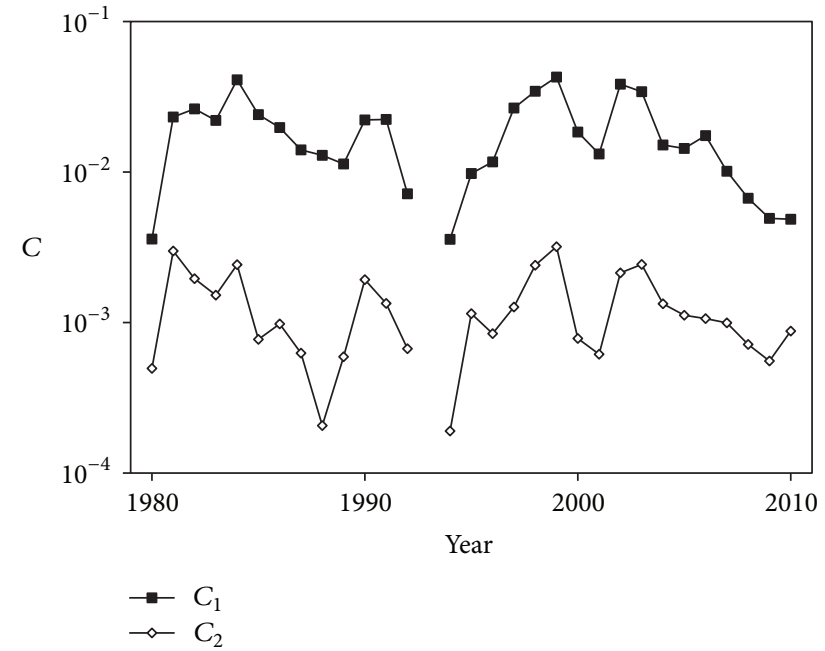

FIgURE 4: Time evolution of parameters $\widetilde{C}_{i}, i=1,2$, of the DPLs corresponding to terrorist attacks over the period 1980-2010, $Y_{r}$.

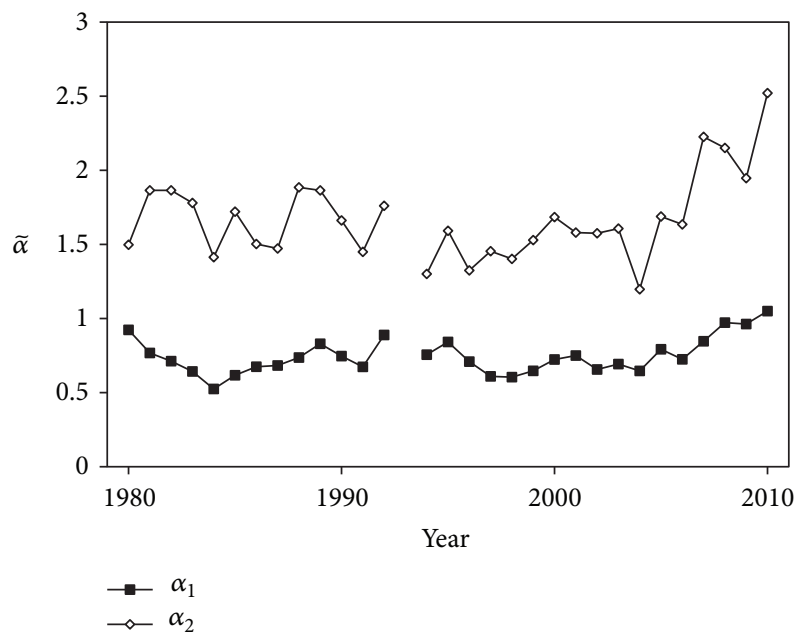

FIGURE 5: Time evolution of parameters $\widetilde{\alpha}_{i}, i=1,2$, of the DPLs corresponding to terrorist attacks over the period 1980-2010, $Y_{r}$.

Bombing/Explosion, Facility/Infrastructure Attack, Hijacking, Hostage Taking (Barricade Incident), Hostage Taking (Kidnapping), and Unarmed Assault $\}$.

Most cases are characterized by DPLs. However, in a few situations a SPL fits better to the data. The main results are summarized in Table 1. Moreover, we observed that the parameters corresponding to all distributions characterized by DPLs display a pattern similar to the ones mentioned previously (Figures 3 and 6 ), where the lines connecting the slopes and intercepts of DPL1 $\left(\widetilde{\alpha}_{1}, \widetilde{C}_{1}\right)$ to its companion DPL2 $\left(\widetilde{\alpha}_{2}, \widetilde{C}_{2}\right)$, for the same data set, have identical orientation in the $(\widetilde{C}, \widetilde{\alpha})$ Cartesian space.

\section{Entropy of Catastrophic Phenomena}

In this section we analyse the entropy of data collected from the GTD database, that is, data related to terrorism. To

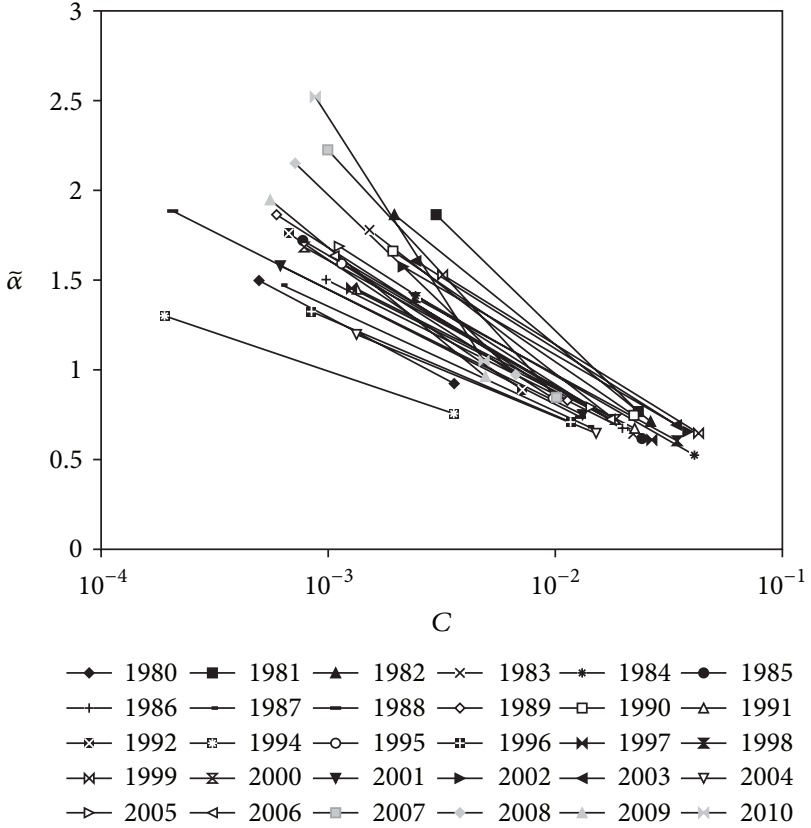

Figure 6: Locus of the parameters $\left(\widetilde{C}_{i}, \widetilde{\alpha}\right), i=1,2$ that characterize the distributions of terrorist attacks over the period 1980-2010, $Y_{r}$.

calculate the entropies we construct histograms of relative frequencies, using bins of width one (one casualty), and approximate the probabilities $p_{i}$ by the relative frequencies. We present results obtained for terrorist events grouped by year $\left(Y_{r}\right)$, as defined in the previous section. Nevertheless, it should be noticed that similar results are obtained for all other human-made and natural hazards.

Clausius [37] and Boltzmann [38] were the first authors to define entropy in the field of thermodynamics. Later on, Shannon [39] and Jaynes [40] applied their results to information theory [41].

The most celebrated entropy is the so-called Shannon entropy $S$ defined by

$$
S=-\sum_{i=1}^{W} p_{i} \ln p_{i}
$$

The Shannon entropy represents the expected value of the information $-\ln p_{i}$. Therefore, for the uniform probability distribution we have $p_{i}=W^{-1}$ and the Shannon entropy takes its maximum value $S=\ln W$, yielding the Boltzmann's famous formula, up to a multiplicative factor $k$ denoting the Boltzmann constant. Thus, in thermodynamic equilibrium, the Shannon entropy can be identified as the "physical entropy" of the system.

Rényi and Tsallis entropies are generalizations of Shannon's entropy and are given by, respectively,

$$
\begin{gathered}
S_{q}^{(R)}=\frac{1}{1-q} \ln \left(\sum_{i=1}^{W} p_{i}^{q}\right), \quad q>0, \\
S_{q}^{(T)}=\frac{1}{q-1}\left(1-\sum_{i=1}^{W} p_{i}^{q}\right) .
\end{gathered}
$$


TABLE 1: PL fit to the distributions of casualties in terrorist attacks.

\begin{tabular}{|c|c|c|c|c|}
\hline Criterion & Category & $\begin{array}{c}\text { SPL } \\
(\widetilde{C}, \widetilde{\alpha})\end{array}$ & $\begin{array}{c}\text { DPL1 } \\
\left(\widetilde{C}_{1}, \widetilde{\alpha}_{1}\right) \\
\end{array}$ & $\begin{array}{c}\text { DPL2 } \\
\left(\widetilde{C}_{2}, \widetilde{\alpha}_{2}\right)\end{array}$ \\
\hline \multirow{6}{*}{ Weapon $\left(W_{i}\right)$} & $W_{1}$ & 0.03910 .5849 & & \\
\hline & $W_{2}$ & & 0.00820 .7514 & 0.00021 .7198 \\
\hline & $W_{3}$ & & 0.00170 .8660 & 0.00001 .9624 \\
\hline & $W_{4}$ & & 0.02360 .6877 & 0.00211 .4890 \\
\hline & $W_{5}$ & & 0.00570 .6909 & 0.00031 .3579 \\
\hline & $W_{6}$ & 0.02490 .7801 & & \\
\hline \multirow{13}{*}{ Region $\left(R_{i}\right)$} & $R_{1}$ & 0.04381 .0147 & & \\
\hline & $R_{2}$ & & 0.02140 .6412 & 0.00091 .6905 \\
\hline & $R_{3}$ & 0.01741 .1905 & & \\
\hline & $R_{4}$ & 0.00650 .9073 & & \\
\hline & $R_{5}$ & 0.00121 .2141 & & \\
\hline & $R_{6}$ & & 0.00910 .8051 & 0.00061 .7088 \\
\hline & $R_{7}$ & 0.00091 .1415 & & \\
\hline & $R_{8}$ & & 0.01930 .7814 & 0.00082 .0675 \\
\hline & $R_{9}$ & & 0.00360 .8742 & 0.00012 .0552 \\
\hline & $R_{10}$ & & 0.01280 .8561 & 0.00121 .6578 \\
\hline & $R_{11}$ & & 0.00910 .6454 & 0.00041 .3132 \\
\hline & $R_{12}$ & 0.00230 .9956 & & \\
\hline & $R_{13}$ & 0.00011 .5523 & & \\
\hline \multirow{19}{*}{ Target $\left(T_{i}\right)$} & $T_{1}$ & & 0.04380 .5144 & 0.00921 .2488 \\
\hline & $T_{2}$ & & 0.00570 .8884 & 0.00031 .7180 \\
\hline & $T_{3}$ & & 0.00021 .3589 & 0.00120 .8458 \\
\hline & $T_{4}$ & 0.04520 .7923 & & \\
\hline & $T_{5}$ & 0.00151 .1259 & & \\
\hline & $T_{6}$ & & 0.00061 .1152 & 0.00011 .5162 \\
\hline & $T_{7}$ & 0.00141 .6106 & & \\
\hline & $T_{8}$ & & 0.05760 .4694 & 0.00940 .9070 \\
\hline & $T_{9}$ & & 0.03530 .5669 & 0.00091 .6291 \\
\hline & $T_{10}$ & & 0.00900 .9645 & 0.00311 .2573 \\
\hline & $T_{11}$ & & 0.00470 .8991 & 0.00011 .8354 \\
\hline & $T_{12}$ & & 0.00360 .7778 & 0.00001 .8667 \\
\hline & $T_{13}$ & & 0.01580 .7353 & 0.00181 .6106 \\
\hline & $T_{14}$ & 0.04620 .9774 & & \\
\hline & $T_{15}$ & & 0.01680 .8610 & 0.00261 .6824 \\
\hline & $T_{16}$ & 0.02340 .7635 & & \\
\hline & $T_{17}$ & & 0.03400 .5892 & 0.00141 .5178 \\
\hline & $T_{18}$ & & 0.02250 .7389 & 0.00741 .0708 \\
\hline & $T_{19}$ & & 0.01080 .7918 & 0.00251 .1650 \\
\hline \multirow{8}{*}{ Type $\left(A_{i}\right)$} & $A_{1}$ & & 0.00490 .7346 & 0.00001 .7982 \\
\hline & $A_{2}$ & & 0.00141 .5201 & 0.00012 .6555 \\
\hline & $A_{3}$ & & 0.00590 .8116 & 0.00012 .0621 \\
\hline & $A_{4}$ & & 0.02610 .6339 & 0.00161 .4880 \\
\hline & $A_{5}$ & 0.03050 .7186 & & \\
\hline & $A_{6}$ & 0.01160 .7316 & & \\
\hline & $A_{7}$ & & 0.00720 .9689 & 0.00141 .6214 \\
\hline & $A_{8}$ & & 0.04710 .7006 & 0.00342 .0186 \\
\hline
\end{tabular}




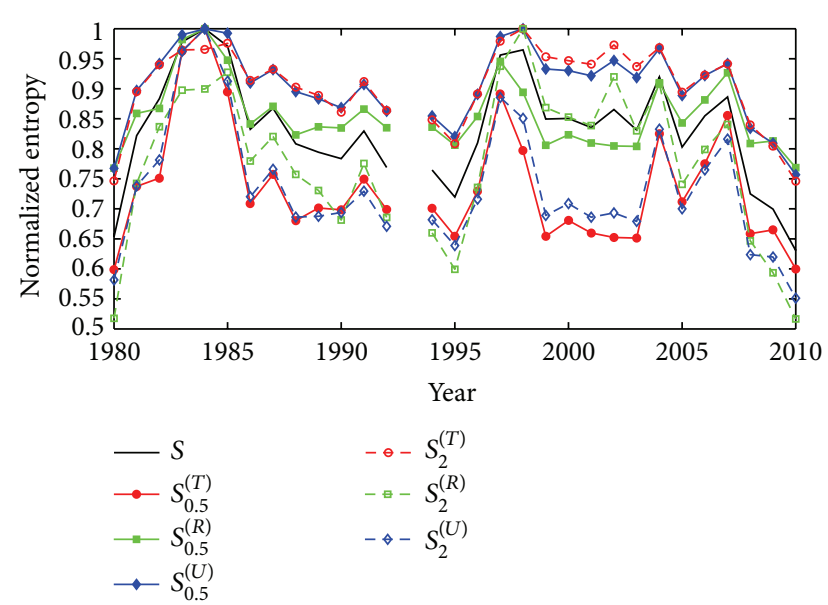

FIgure 7: Total Shannon, Tsallis, Rényi, and Ubriaco normalized entropies, as a function of the year of the events, $Y_{r} ; q=0.5$ and $q=2$.

Tsallis entropy reduces to Rényi entropy when $q \rightarrow 1$. Tsallis entropy was applied to diffusion equations [42] and FokkerPlanck systems [43]. Rényi entropy has an inverse power law equilibrium distribution [44] and satisfies the zeroth law of thermodynamics [45]. The two parameters Sharma-Mittal entropy [46] is accepted as a generalization of Tsallis, Rényi, and Boltzmann-Gibbs entropies, for limiting cases of the parameters [47].

Recently, more general entropy measures have been proposed in the literature, where the additivity axiom has been relaxed. For instance, Ubriaco [48] proposed the following formula for the fractional entropy:

$$
S_{q}^{(U)}=\sum_{i=1}^{W}\left(-\ln p_{i}\right)^{q} p_{i}
$$

that has the same properties as the Shannon entropy except additivity.

Applications of entropy in distinct complex systems can be found in [49-57].

In Figure 7 the total Shannon normalized entropy $(S)$ is depicted, as well as Tsallis' $\left(S_{q}^{(T)}\right)$, Rényi's $\left(S_{q}^{(R)}\right)$ and Ubriaco's $\left(S_{q}^{(U)}\right)$, for $q=0.5$, and $q=2$, as a function of the year of the events, $Y_{r}$. Figures 9,10 , and 11 show $S_{q}^{(T)}, S_{q}^{(R)}$ and $S_{q}^{(U)}$ normalized entropies, as a function of the year, $Y_{r}$, and entropy parameter $0.1 \leq q \leq 10$.

In Figure 7 we observe two types of behavior, namely, short- and long-term phenomena. In what concerns short time behavior, we verify peaks during 1983-1985, 1997-1998, 2004-2007, and minima at 1980, 1995, and 2010. In what concerns long-time relationships a smooth decreasing is observed for $S_{0.5}^{(T)}, S_{0.5}^{(R)}$, and $S_{2}^{(U)}$. Removing the maxima and minima we get a time series for years 1981, 1982, 19861992, 1994-1996, 1999, 2000, 2002, 2003, 2008, and 2009 (Figure 8). Larger/smaller entropies correspond to charts closer/afar uniform distributions; therefore, seemingly, we have less/more organized terrorist events in global terms.

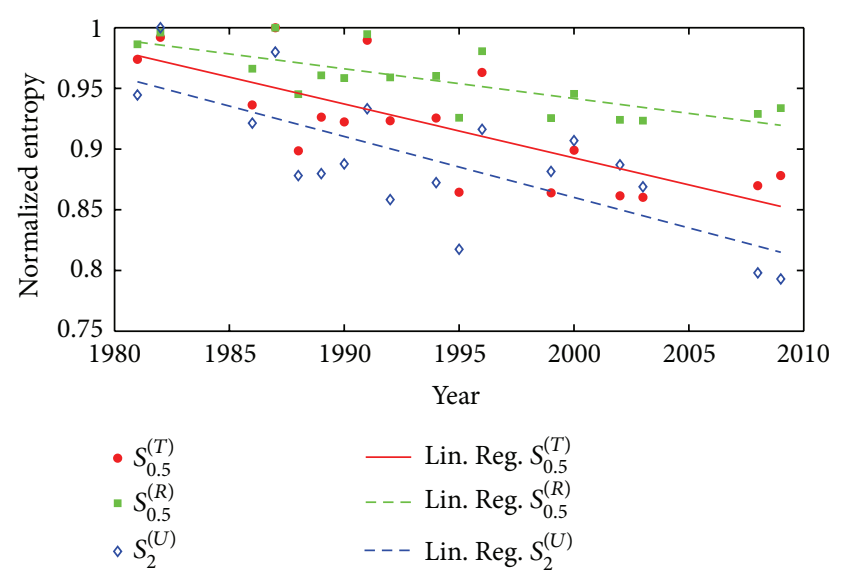

Figure 8: Total Tsallis and Rényi normalized entropies $(q=0.5)$ and Ubriaco's $(q=2)$, as a function of the events in years $\{1981,1982$, 1986-1992, 1994-1996, 1999, 2000, 2002, 2003, 2008, 2009\}.

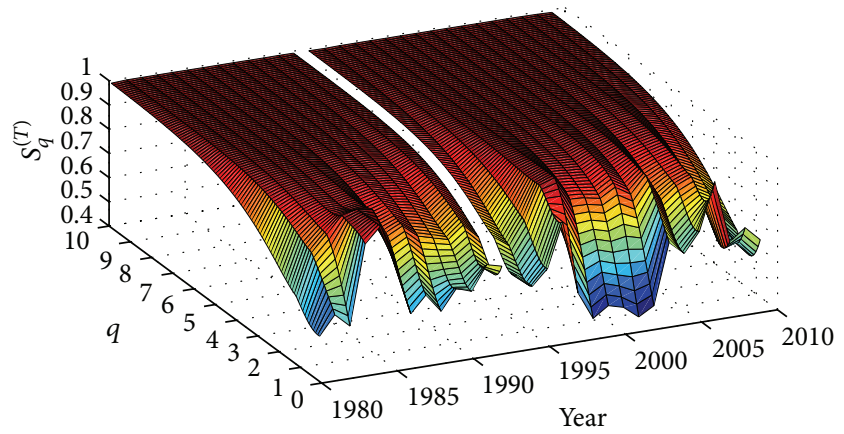

FIgURE 9: Total Tsallis normalized entropy, $S_{q}^{(T)}$, as a function of the year of the events, $Y_{r}$, and parameter $q$.

These conclusions remain invariant for Figures 9 to 11, where we vary both the entropy definition and the parameter tuning. Therefore, we conclude that such results are robust against such type of variations.

As discussed in the previous section, the statistical distributions of real data can be approximated by either single or double PLs. In the latter case, we study, not only the total entropy, but also the entropy associated to each part of the distributions. Therefore, we compute the entropy associated to DPL1 and DPL2, that approximate the first and second part of the distributions, respectively. When adopting this procedure we are restricted to the Shannon and Ubriaco entropies, as Tsallis' and Rényi's do not admit the associativity described above.

In the sequel we present several results of the analysis, taking into account the grouping criteria $Y_{r}, W_{i}, R_{j}, T_{k}$, and $A_{l}$. Figure 12 depicts the Shannon entropy versus parameter $\widetilde{\alpha}$ for all statistical distributions. The black squares (denoted "SPL") correspond to the plot of $\widetilde{\alpha}$ versus the total entropy of the respective distributions, $S$. The black circles (denoted "DPL1") are the plot of $\widetilde{\alpha}_{1}$ versus the entropy associated to the first parts of the distributions, $S_{1}$. The white circles (denoted "DPL2") represent the plot of $\widetilde{\alpha}_{2}$ versus the entropy associated 


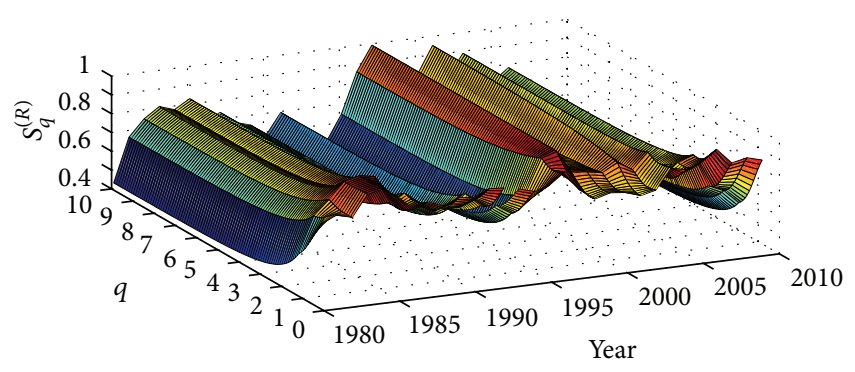

FIGURE 10: Total Rényi normalized entropy, $S_{q}^{(R)}$, as a function of the year of the events, $Y_{r}$, and parameter $q$.

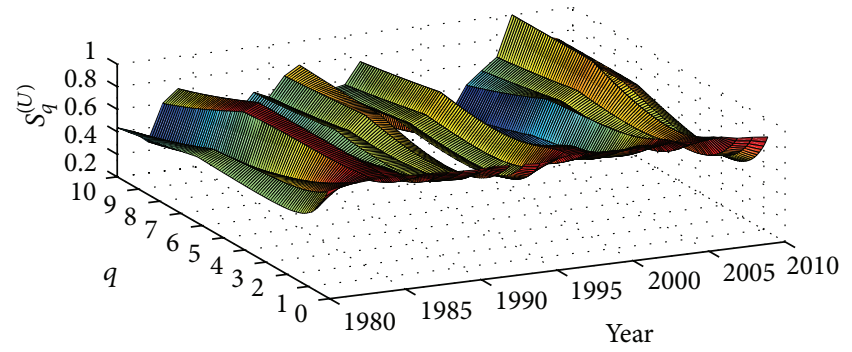

FIGURE 11: Total Ubriaco normalized entropy, $S_{q}^{(U)}$, as a function of the year of the events, $Y_{r}$, and parameter $q$.

to the second parts of the distributions, $S_{2}$. As can be seen, for the distributions that behave as single PLs, higher entropies correspond to the lower values of the parameter $\widetilde{\alpha}$ and the two parameters are linearly related. A similar pattern is observed for the parameters corresponding to DPL1. For DPL2 the parameter $\widetilde{\alpha}_{2}$ increases with entropy, but the almost linear relation between both parameters remains.

Figure 13 shows identical results for the Ubriaco entropy. The plot corresponds to $q=0.5$, nevertheless, identical results are obtained for other values.

For all DPLs related to terrorist events, $n(n=1, \ldots, 60)$, we find that the parameters $\left(\widetilde{C}_{i}, \widetilde{\alpha}_{i}\right), i=1,2$ obey the following relation:

$$
\widetilde{\alpha}_{1 n}\left|\log \widetilde{C}_{1 n}\right|^{p}=\widetilde{\alpha}_{2 n}\left|\log C_{2 n}\right|^{p}+\epsilon_{n},
$$

where $p=-1.612$. The mean value of $\epsilon_{n}$ is $\bar{\epsilon}=0.002$, and the corresponding standard deviation is $\sigma_{\epsilon}=0.036$. Moreover, for the analyzed data, we find $\widetilde{\alpha}_{i n}\left|\log \widetilde{C}_{i n}\right|^{p}=k_{i n}$. Parameters $k_{\text {in }}$ are approximately constant, with mean value $\bar{k}=0.277$ and standard deviation $\sigma_{k}=0.06$.

It is worth noticing that (5) is similar to Poisson's law of an adiabatic reversible process, involving ideal gases, given by

$$
P_{1} V_{1}^{\gamma}=P_{2} V_{2}^{\gamma}
$$

where variables $P_{i}$ and $V_{i}$ represent pressure and specific volume, respectively. Equation (6) implies that $P V^{\gamma}=$ const. Parameter $\gamma$ is called Poisson's coefficient, taking values $\gamma=$ $5 / 3 \simeq 1.67$ and $\gamma=7 / 5=1.40$ for monoatomic and diatomic gases, respectively. Additionally, it should be noticed that the absolute value of the exponent $p$ is very similar to the one observed for an ideal gas undergoing a reversible adiabatic process.

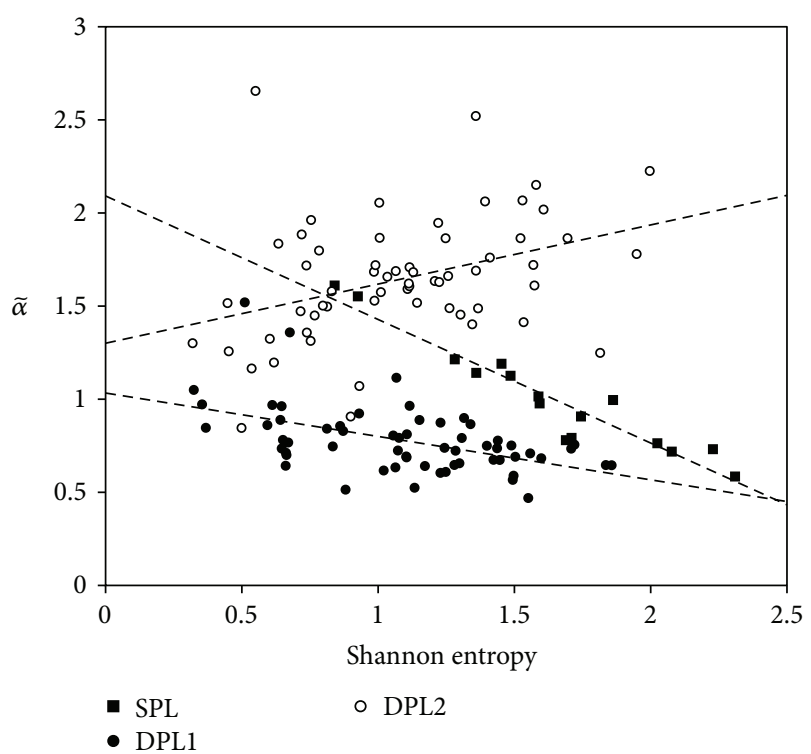

FIGURE 12: Parameters $\tilde{\alpha}$ versus Shannon entropies for the distributions corresponding to the criteria $Y_{r}, W_{i}, R_{j}, T_{k}$, and $A_{l}$; "SPL" corresponds to $(S, \widetilde{\alpha})$; "DPL1" corresponds to $\left(S_{1}, \widetilde{\alpha}_{1}\right)$; "DPL2" corresponds to $\left(S_{2}, \widetilde{\alpha}_{2}\right)$.

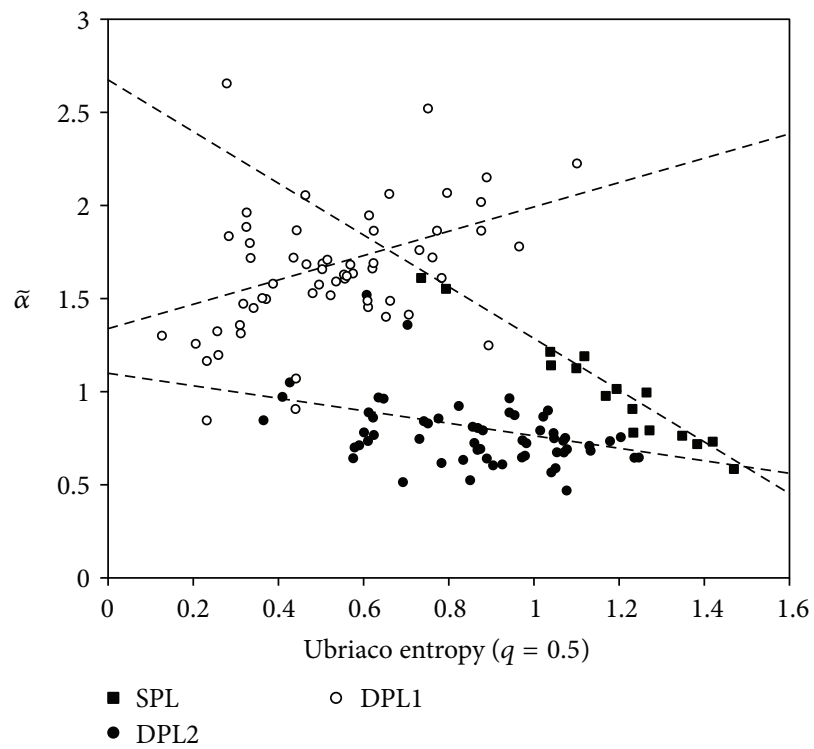

FIGURE 13: Parameters $\widetilde{\alpha}$ versus Ubriaco entropies for the distributions corresponding to the criteria $Y_{r}, W_{i}, R_{j}, T_{k}$, and $A_{l}$; "SPL" corresponds to $\left(S_{q}^{(U)}, \widetilde{\alpha}\right)$; "DPL1" corresponds to $\left(S_{q 1}^{(U)}, \widetilde{\alpha}_{1}\right)$; "DPL2" corresponds to $\left(S_{q 2}^{(U)}, \widetilde{\alpha}_{2}\right) ; q=0.5$.

\section{Conclusions}

PLs have been widely reported in the modeling of distinct phenomena and have been associated with long memory behavior, self-similarity, fractal structures, and fractional calculus.

In this paper we reviewed interesting and important results on PLs distributions and their applications to the 
modeling of the number of victims in catastrophic events. We found double PL behavior in real data of catastrophic occurrences, in particular, terrorism. We have plotted the two PLs parameters, $\left(\widetilde{C}_{i}, \widetilde{\alpha}_{i}\right), i=1,2$, corresponding to certain events, and observed an interesting pattern in the chart, where the lines that connect each pair of points defining the double PLs are almost aligned to each other. We have also computed the entropy of the data sets. This complementary analysis of the numerical data revealed extra relationships but the fact is that these phenomena have a dense and rich volume of characteristics and further research efforts are needed to a deeper understanding.

\section{References}

[1] J. Tenreiro Machado, F. B. Duarte, and G. M. Duarte, "Power law analysis of financial index dynamics," Discrete Dynamics in Nature and Society, vol. 2012, Article ID 120518, 12 pages, 2012.

[2] J. Tenreiro Machado, "And I say to myself: 'What a fractional worl," Fractional Calculus and Applied Analysis, vol. 14, no. 4, pp. 635-654, 2011.

[3] E. Scalas, R. Gorenflo, and F. Mainardi, "Fractional calculus and continuous-time finance," Physica A, vol. 284, no. 1-4, pp. 376$384,2000$.

[4] F. Mainardi, M. Raberto, R. Gorenflo, and E. Scalas, "Fractional calculus and continuous-time finance. II: the waiting-time distribution," Physica A, vol. 287, no. 3-4, pp. 468-481, 2000.

[5] B. J. West, "Fractal physiology and the fractional calculus: a perspective," Frontiers in Physiology, vol. 1, p. 12, 2010.

[6] B. Gutenberg and R. F. Richter, "Frequency of earthquakes in california," Bulletin of the Seismological Society of America, vol. 34, pp. 185-188, 1944.

[7] L. F. Richardson, "Variation of the Frequency of Fatal Quarrels with Magnitude," Journal of the American Statistical Association, vol. 43, pp. 523-546, 1948.

[8] J. M. Carlson and J. S. Langer, "Mechanical model of an earthquake fault," Physical Review A, vol. 40, no. 11, pp. 6470-6484, 1989.

[9] D. C. Roberts and D. L. Turcotte, "Fractality and self-organized criticality of wars," Fractals, vol. 6, no. 4, pp. 351-357, 1998.

[10] D. R. Davis and D. E. Weinstein, "Bones, bombs, and break points: the geography of economic activity," The American Economic Review, vol. 92, no. 5, pp. 1269-1289, 2002.

[11] L. E. Cederman, "Modeling the size of wars: from billiard balls to sandpiles," American Political Science Review, vol. 97, no. 1, pp. 135-150, 2003.

[12] J. C. Bohorquez, S. Gourley, A. R. Dixon, M. Spagat, and N. F. Johnson, "Common ecology quantifies human insurgency," Nature, vol. 462, no. 7275, pp. 911-914, 2009.

[13] V. Pareto, Cours d'Economie Politique, Droz, Geneva, Switzerland, 1896.

[14] F. Auerbach, "Das Gesetz der Belvolkerungskoncentration," Petermanns Geographische Mitteilungen, vol. 59, pp. 74-76, 1913.

[15] J. B. Estoup, Gammes Stenographiques, Institut de France, 1916.

[16] G. Zipf, Selective Studies and the Principle of Relative Frequency in Language, Harvard University Press, Cambridge, Mass, USA, 1932.

[17] G. Zipf, Human Behavior and the Priciple of Least Effort, Addison-Wesley, Cambridge, Mass, USA, 1949.

[18] W. Li, "References on Zipf's law," http://www.sorinsolomon .net/ sorin/ccs/zipf/references\%20on\%20zipf\%27s\%20law.htm.
[19] D. Sornette, Critical Phenomena in Natural Sciences, chapter 14, Springer, Heidelberg, Germany, 2nd edition, 2003.

[20] C. M. A. Pinto, A. M. Lopes, and J. A. Tenreiro Machado, "A review of power laws in real life phenomena," Communications in Nonlinear Science and Numerical Simulations, vol. 17, no. 9, pp. 3558-3578, 2012.

[21] J. Tuldava, "The frequency spectrum of text and vocabulary," Journal of Quantitative Linguistics, vol. 3, no. 1, pp. 38-50, 1996.

[22] G. Martínez-Mekler, R. Alvarez Martínez, M. Beltrán del Río, R. Mansilla, P. Miramontes, and G. Cocho, "Universality of rankordering distributions in the arts and sciences," PLoS One, vol. 4, no. 3, Article ID e4791, 2009.

[23] R. F. I. Cancho and R. V. Solé, "Two regimes in the frequency of words and the origins of complex lexicons: Zipf's law revisited," Journal of Quantitative Linguistics, vol. 8, no. 3, pp. 165-173, 2001.

[24] R. Alvarez-Martinez, G. Martinez-Mekler, and G. Cochoa, "Order-disorder transition in conflicting dynamics leading to rank-frequency generalized beta distributions," Physica A, vol. 390, no. 1, pp. 120-130, 2011.

[25] L. F. Richardson, Statistics of Deadly Quarrels, Quadrangle Books, Chicago, Ill, USA, 1960.

[26] F. Guzzetti, "Landslide fatalities and the evaluation of landslide risk in Italy, Engineering Geology, vol. 58, no. 2, pp. 89-107, 2000.

[27] S. N. Jonkman, "Global perspectives on loss of human life caused by floods," Natural Hazards, vol. 34, no. 2, pp. 151-175, 2005.

[28] N. F. Johnson, M. Spagat, J. A. Restrepo et al., "Universal patterns underlying ongoingwars and terrorism," http://arxiv.org/ abs/physics/0605035.

[29] D. S. Geller and J. David Singer, Nations at War: A Scientific Study of International Conflict, Cambridge Studies in International Relations, Cambridge University Press, Cambridge, UK, 1998.

[30] O. Becerra, N. Johnson, P. Meier, J. Restrepo, and M. Spagat, "Natural disasters, casualties and power laws: a comparative analysis with armed conflict," in Proceedings of the Annual Meeting of the American Political Science Association, Loews Philadelphia, and the Pennsylvania Convention Center, Philadelphia, Pa, USA, 2006, http://www.allacademic.com/ meta/p151714_index.html.

[31] M. E. J. Newman, "Power laws Pareto distributions and Zipf's law," Contemporary Physics, vol. 46, pp. 323-351, 2005.

[32] A. Clauset and M. Young, "Scale invariance in global terrorism," http://arxiv.org/abs/physics/0502014.

[33] K. T. Bogen and E. D. Jones, "Risks of mortality and morbidity from worldwide terrorism: 1968-2004," Risk Analysis, vol. 26, no. 1, pp. 45-59, 2006.

[34] A. Clauset, M. Young, and K. S. Gleditsch, "On the frequency of severe terrorist events," Journal of Conflict Resolution, vol. 51, no. 1, pp. 58-87, 2007.

[35] EM-DAT, "The OFDA/CRED International Disaster Database," Université catholique de Louvain, Brussels, Belgium, http:// www.emdat.net/.

[36] National Consortium for the Study of Terrorism and Responses to Terrorism (START), "Global Terrorism Database," 2011, http://www.start.umd.edu/gtd/.

[37] R. Clausius, The Mechanical Theory of Heat with Its Applications to the Steam Engine and to Physical Properties of Bodies, John van Voorst, London, UK, 1865.

[38] L. Boltzmann, Vorlesungen ber Gastheorie, J. A. Barth, Leipzig, Germany, 1896. 
[39] C. E. Shannon, "A mathematical theory of communication," The Bell System Technical Journal, vol. 27, pp. 379-423, 623-656, 1948.

[40] E. T. Jaynes, "Information theory and statistical mechanics," vol. 106, pp. 620-630, 1957.

[41] A. I. Khinchin, Mathematical Foundations of Information Theory, Dover, New York, NY, USA, 1957.

[42] A. R. Plastino, M. Casas, and A. Plastino, "A nonextensive maximum entropy approach to a family of nonlinear reactiondiffusion equations," Physica A, vol. 280, no. 3, pp. 289-303, 2000.

[43] T. D. Frank and A. Daffertshofer, " $H$-theorem for nonlinear Fokker-Planck equations related to generalized thermostatistics," Physica A, vol. 295, no. 3-4, pp. 455-474, 2001.

[44] E. K. Lenzi, R. S. Mendes, and L. R. da Silva, "Statistical mechanics based on Renyi entropy," Physica A, vol. 280, no. 3-4, pp. 337$345,2000$.

[45] A. S. Parvan and T. S. Biró, "Extensive Rényi statistics from nonextensive entropy," Physics Letters A, vol. 340, no. 5-6, pp. 375387, 2005.

[46] B. D. Sharma and D. P. Mittal, "New nonadditive measures of entropy for discrete probability distributions," Journal of Mathematical Sciences, vol. 10, pp. 28-40, 1975.

[47] T. D. Frank and A. Daffertshofer, "Exact time-dependent solutions of the Renyi Fokker-Planck equation and the FokkerPlanck equations related to the entropies proposed by Sharma and Mittal," Physica A, vol. 285, no. 3, pp. 351-366, 2000.

[48] M. R. Ubriaco, "Entropies based on fractional calculus," Physics Letters A, vol. 373, no. 30, pp. 2516-2519, 2009.

[49] P. T. Landsberg and V. Vedral, "Distributions and channel capacities in generalized statistical mechanics," Physics Letters A, vol. 247, no. 3, pp. 211-217, 1998.

[50] A. Plastino and A. R. Plastino, "Tsallis Entropy and Jaynes' information theory formalism," Brazilian Journal of Physics, vol. 29, no. 1, pp. 50-60, 1999.

[51] X. Li, C. Essex, M. Davison, K. H. Hoffmann, and C. Schulzky, "Fractional diffusion, irreversibility and entropy," Journal of Non-Equilibrium Thermodynamics, vol. 28, no. 3, pp. 279-291, 2003.

[52] H. J. Haubold, A. M. Mathai, and R. K. Saxena, "BoltzmannGibbs entropy versus Tsallis entropy: recent contributions to resolving the argument of Einstein concerning "neither Herr Boltzmann nor Herr Planck has given a definition of W"? Essay review," Astrophysics and Space Science, vol. 290, no. 3-4, pp. 241-245, 2004.

[53] P. N. Rathie and S. da Silva, "Shannon, Lévy, and Tsallis: a note," Applied Mathematical Sciences, vol. 2, no. 28, pp. 1359-1363, 2008.

[54] R. M. Gray, Entropy and Information Theory, Springer, New York, NY, USA, 2009.

[55] J. A. Tenreiro Machado, A. C. Costa, and M. F. M. Lima, "Dynamical analysis of compositions," Nonlinear Dynamics, vol. 65, no. 4, pp. 339-412, 2011.

[56] J. A. T. Machado, A. C. Costa, and M. D. Quelhas, "Entropy analysis of the DNA code dynamics in human chromosomes," Computers \& Mathematics with Applications, vol. 62, no. 3, pp. 1612-1617, 2011.

[57] J. A. Tenreiro Machado, A. C. Costa, and M. D. Quelhas, "Shannon, Rényie and Tsallis entropy analysis of DNA using phase plane," Nonlinear Analysis: Real World Applications, vol. 12, no. 6, pp. 3135-3144, 2011. 


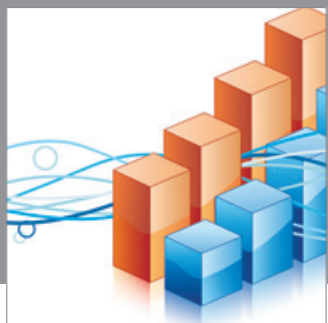

Advances in

Operations Research

mansans

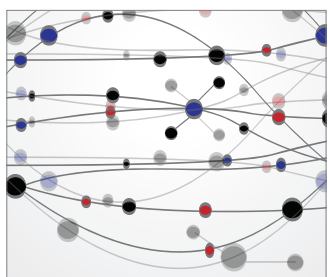

The Scientific World Journal
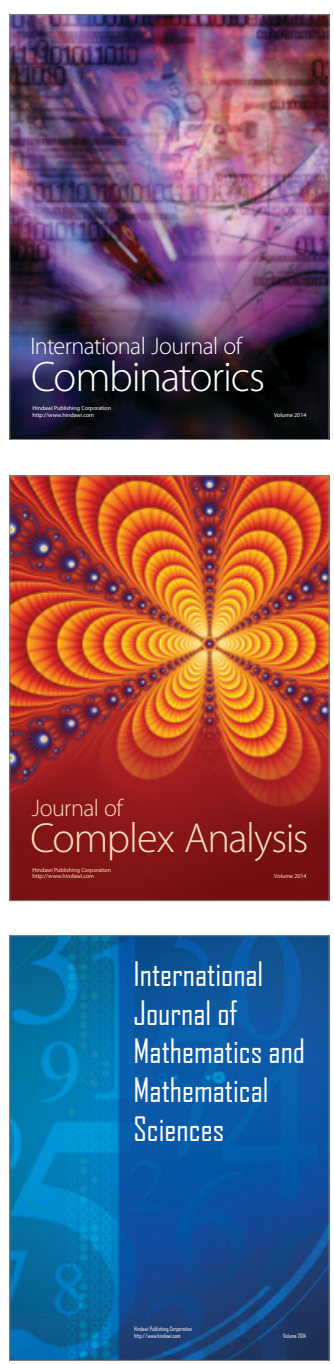
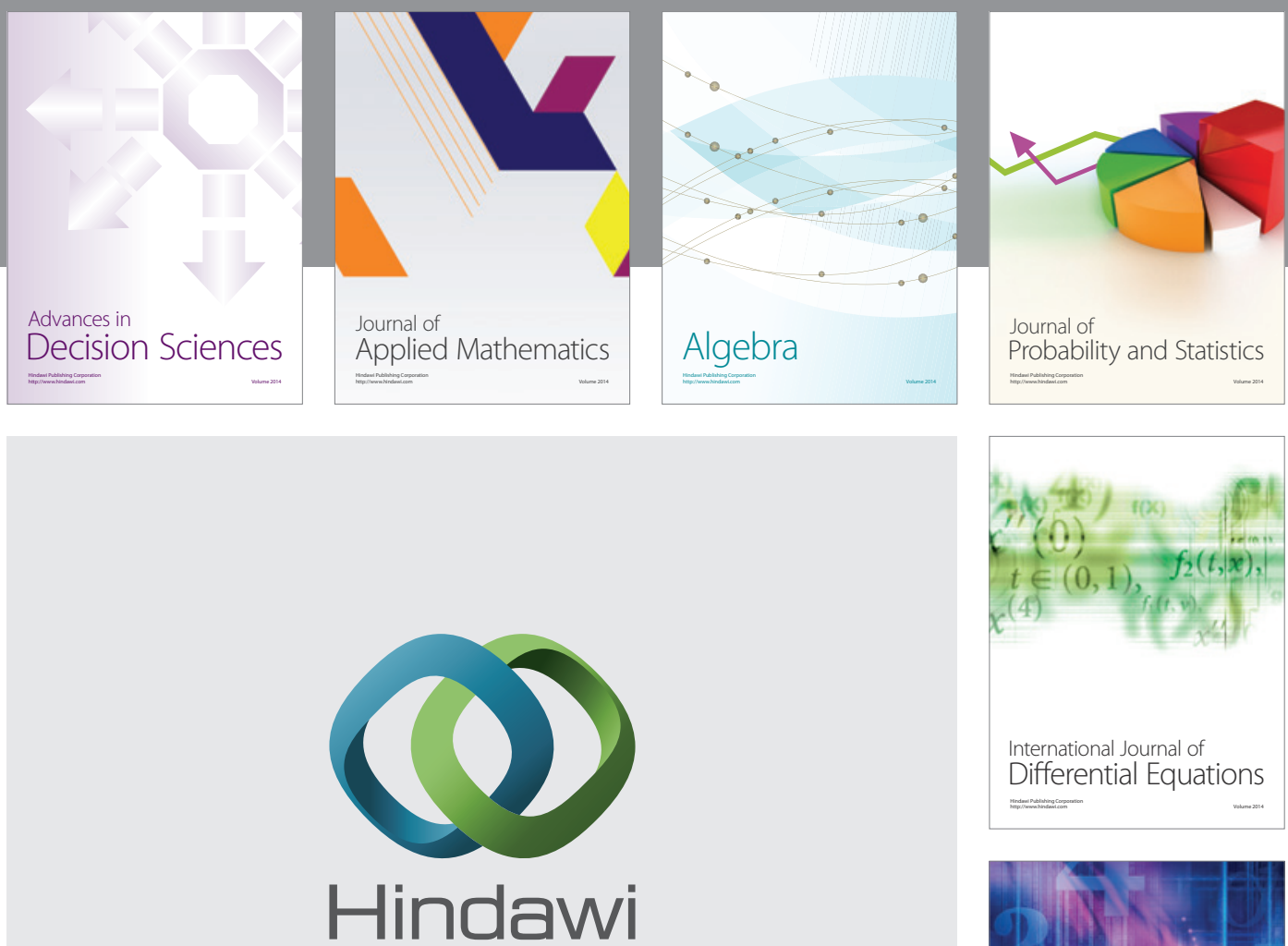

Submit your manuscripts at http://www.hindawi.com
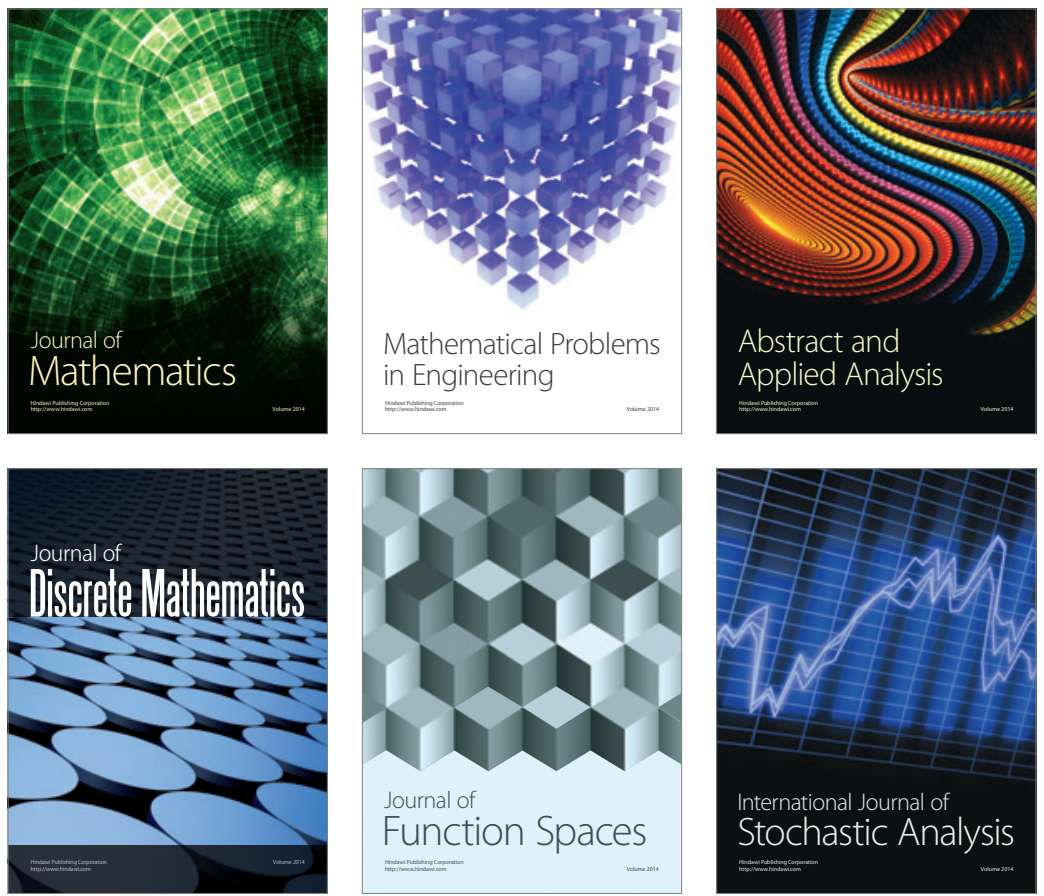

Journal of

Function Spaces

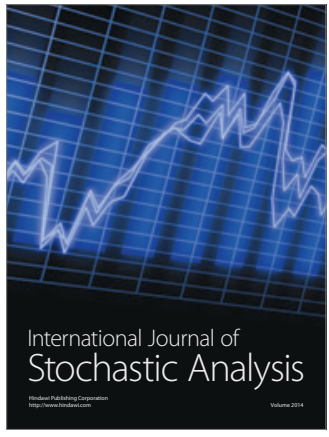

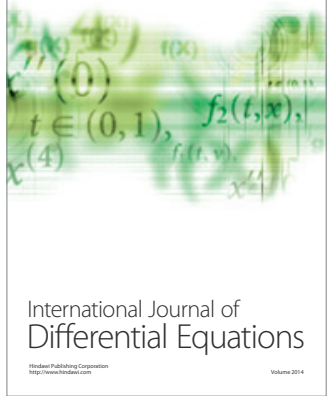
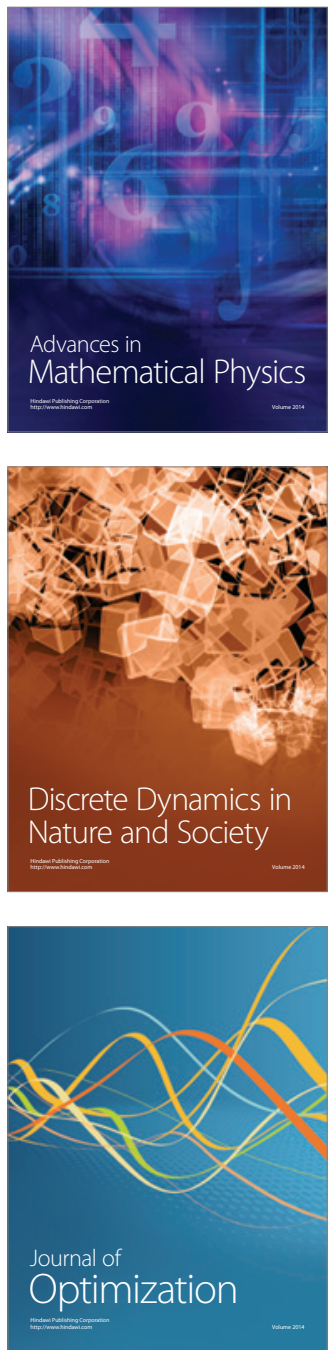